\title{
Surgical Causes of Significant Intraoperative Neuromonitoring Signal Changes in Three-Column Spinal Surgery
}

\author{
Seung Myung Wi ${ }^{1}$, Sang-Min Park ${ }^{2}$, Sam Yeol Chang ${ }^{3}$, Jeongik Lee ${ }^{4}$, \\ Sung-Min $\mathrm{Kim}^{5}$, Bong-Soon Chang ${ }^{3}$, Hyoungmin $\mathrm{Kim}^{3}$ \\ ${ }^{I}$ Department of Orthopedic Surgery, Cheju Halla General Hospital, Jeju, Korea \\ ${ }^{2}$ Department of Orthopedic Surgery, Seoul National University Bundang Hospital, Seoul, Korea \\ ${ }^{3}$ Department of Orthopedic Surgery, Seoul National University Hospital, Seoul, Korea \\ ${ }^{4}$ Department of Orthopedic Surgery, Chung-Ang University Hospital, Seoul, Korea \\ ${ }^{5}$ Department of Neurology, Seoul National University Hospital, Seoul, Korea
}

Study Design: Retrospective case series.

Purpose: To evaluate the risks and causes of neurologic complications in three-column spinal surgery by analyzing intraoperative neurophysiological monitoring (IONM) data.

Overview of Literature: Three-column spinal surgery, which may be required to correct complex spinal deformities or resection of spinal tumors, is known to carry a high risk of neurologic complications. However, few studies reported a specific surgical procedure related to a significant IONM signal change during surgery.

Methods: Multimodality IONM data, including somatosensory-evoked potentials (SSEP) and motor-evoked potentials (MEP), were reviewed in 64 patients who underwent three-column spinal surgery from 2011 to 2015. Surgical procedures included posterior vertebral column resection, pedicle subtraction osteotomy, total en bloc spondylectomy, piecemeal spondylectomy, and corpectomy with laminectomy ( $\mathrm{n}=27$ ) in three cervical, 34 thoracic, and 31 lumbar procedures.

Results: Significant IONM signal changes occurred in 11 of 64 (17.1\%) patients. SSEP and MEP were changed in 11 patients. Postoperative neurologic deterioration occurred in $54.5 \%$ ( 6 of 11) of the patients, and two of them were permanent. There was no postoperative neurologic deterioration in patients without significant signal change. Suspected causes of IONM data changes are as follows: adhesion/tethering, translation, contusion, and perfusion.

Conclusions: Based on the results of this study, to enhance neurologic safety in three-column spinal surgery, surgeons should pay attention to protect the spinal cord from mechanical insult, especially when the spinal column was totally destabilized during surgery, and not to compromise perfusion to the spinal cord in close cooperation with a neurologist and anesthesiologist.

Keywords: Corpectomy; Intraoperative monitoring; Neurophysiologic monitoring; Spondylectomy; Three-column spinal surgery

Received Mar 9, 2021; Revised Jul 13, 2021; Accepted Jul 13, 2021

Corresponding author: Hyoungmin Kim

Department of Orthopedic Surgery, Seoul National University Hospital, 101 Daehak-ro, Jongno-gu, Seoul 03080, Korea

Tel: +82-2-2072-0357, Fax: +82-2-764-2718, E-mail: hmkhm@snu.ac.kr 


\section{Introduction}

The surgical treatment of spinal deformities or tumors continues to evolve with advanced surgical techniques of three-column procedures, such as complex spinal osteotomies, circumferential decompression of the spinal cord, or en bloc segmental resections of the vertebral column. These surgical procedures often present significant neurologic complications. The incidence of neurologic deficits in complex osteotomies was greater in simple spinal surgeries, such as single-level intervertebral fusions, at $0 \%-1 \%$ [1-5]. The reported incidence of neurologic complications during complex osteotomies for spinal deformity correction is $8 \%-27 \%[2,6,7]$. In tumor resection surgeries, the incidence increases from up to $23.8 \%-65.4 \%[1,5,8]$. These incidences represent a significantly higher risk than encountered during conventional spine surgeries, such as simple decompression with or without instrumented posterior fixation.

To improve neurologic safety, intraoperative neurophysiological monitoring (IONM) is used to detect and give a chance to reverse the related causes of neurologic complications during spinal surgery [9-13]. Many studies provide alarm criteria of the IONM signal change and confirm its reliability in detecting neurologic complications [14-17]. However, few of these studies reported a specific surgical procedure related to a significant IONM signal change during surgery. This study performed an indepth analysis of the interplay between the surgical procedure and a significant IONM signal change, especially in three-column spinal surgery, to find a critical surgical step associated with a high risk of neurologic complications during surgery.

\section{Materials and Methods}

The Institutional Review Board of Seoul National University Hospital (IRB approval no., H-1703-156-840) and the Ethics Committee exempted the study for obtaining patient informed consent as this was a minimal risk study. All included surgeries were performed on 64 patients who underwent three-column spinal surgery from October 2011 to November 2015 by two spine-specialist orthopedic surgeons (H.K., B.S.C.) at one institution. Multimodality IONM data, including somatosensory-evoked potentials (SSEP) and motor-evoked potentials (MEP), were reviewed. Surgical procedures involving three col-
Table 1. Demographics of entire cohort

\begin{tabular}{|c|c|}
\hline Characteristic & Value \\
\hline Age (yr) & $51.8(4-80)$ \\
\hline \multicolumn{2}{|l|}{ Sex } \\
\hline Male & 37 \\
\hline Female & 27 \\
\hline \multicolumn{2}{|l|}{ Diagnosis (cases) } \\
\hline Congenital kyphoscoliosis & 8 \\
\hline Primary tumors & 8 \\
\hline Metastasis & 34 \\
\hline Adult deformity & 7 \\
\hline Trauma & 5 \\
\hline Infection & 2 \\
\hline \multicolumn{2}{|l|}{ Surgical procedure (cases) } \\
\hline Total en bloc spondylectomy & 12 \\
\hline Piecemeal spondylectomy & 10 \\
\hline VCR & 7 \\
\hline PSO & 8 \\
\hline Corpectomy with laminectomy & 27 \\
\hline \multicolumn{2}{|l|}{ Level (cases) } \\
\hline Cervical & 3 \\
\hline Thoracic & 34 \\
\hline Lumbar & 31 \\
\hline
\end{tabular}

Values are presented as mean (range) or number.

VCR, vertebral column resection; PSO, pedicle subtraction osteotomy

umns included vertebral column resection $(n=7)$, pedicle subtraction osteotomy $(\mathrm{n}=8)$, total en bloc segmental resection, such as spondylectomy and sagittal resection $(\mathrm{n}=12)$, piecemeal spondylectomy $(\mathrm{n}=10)$, and corpectomy via laminectomy with or without costotransversectomy $(\mathrm{n}=27)$. There were three cervical, 34 thoracic, and 31 lumbar levels. There were 27 females and 37 males. The mean age was 51.8 years. The diagnoses of the operated patients were congenital kyphoscoliosis $(n=8)$, primary spinal tumor $(n=8)$, metastatic spinal tumor $(n=34)$, adult spinal deformity $(n=7)$, trauma $(n=5)$, and infection $(n=2)$ (Table 1). The postoperative neurologic status of all included patients was followed for 36 at least months postoperatively. Patients received intravenous anesthetic agents $(4.5 \mu \mathrm{g} / \mathrm{mL}$ propofol and $1.7 \mathrm{ng} / \mathrm{mL}$ remifentanil) compatible with IONM, and short-acting muscle relaxants were used for intubation but not during surgery. IONM was performed by an electroneurophysiology technologist under the supervision of a neurologist using 
the NIM-ECLIPSE Spinal System (Medtronic Sofamor Danek, Memphis, TN, USA) and following a set of monitoring protocols. MEP recording followed the application of transcranial electric stimulation. Transcranial anodal motor cortex stimulation was performed via electrical pulses through needle electrodes inserted over the C3' and C4' (1-2 cm anterior to $\mathrm{C} 3$ and $\mathrm{C} 4$ positions of the international 10-20 system). Trains of five biphasic pulses with an interstimulus interval of 1-2 ms were delivered at a rate of $1 \mathrm{~Hz}$. Stimulation intensity ranged from 200 to $350 \mathrm{~V}$. Monitored muscles were flexibly determined depending on the operation's spinal level but generally included bilateral deltoid, biceps, thenar, tibialis anterior, gastrocnemius, and abductor halluces. Intraoperative real-time monitoring of electromyogram (EMG) was also performed at the same locations as those used in MEP monitoring. SSEPs were obtained by stimulating the median nerve at the wrist and the posterior tibial nerve at the ankle. A $200 \mu$ s square-wave electrical pulse was presented sequentially to the bilateral median and posterior tibial nerves at a rate of $2.31 \mathrm{~Hz}$ with stimulus intensity ranging from 20 to $40 \mathrm{~mA}$. SSEP was recorded from needle electrodes placed on the scalp at locations $\mathrm{C} 3$ and $\mathrm{C} 4$ and $\mathrm{Cz}$ referenced to Fpz. The upper or lower extremities were selectively monitored depending on the operation's spinal level. Baseline SSEP and MEP were obtained after anesthesia induction but before performing the surgical procedure. Peak-to-peak SSEP amplitude and latency were continuously monitored. Because MEPs provoke patient movement, trials were conducted only after confirmation or when requested during surgery. The request of MEP provocation was made by a surgeon in any recognition of potential insult to neurologic structures during surgery. Significant IONM signal changes were identified based on previously reported criteria. The criteria used to indicate an abnormal SSEP were either a $>50 \%$ decrease in peakto-peak amplitude or a $>10 \%$ increase in latency compared to the baseline. A reduction of more than $50 \%$ in MEP baseline amplitude elicited by direct cortical stimulation was considered a significant change. Relevant EMG activities reported to the surgeon included spikes, bursts, and trains [2,5,15-17]. Abnormal monitoring results were reported immediately by the electroneurophysiologist to allow the surgeon to suspend the surgery and investigate the cause in real-time. Technical and anesthetic variables were assessed and subsequently excluded as the cause of the significant change. Surgical variables, such as defor- mity correction and neural tissue adhesion or tethering, were examined. Corrective steps were performed when abnormal findings were found in these variables. Every event of a significant signal change was documented on the IONM record of each case in the context of surgical procedure and with comments on the suspicious cause through discussion with surgeons and neurologists.

The neurologic evaluation of each patient was conducted before surgery, within 24 hours postoperatively, and 36 months after surgery. The strength of the 10 key muscles ranged from 0 to 5 . A follow-up was conducted up to 36 months after surgery in the group with an immediate postoperative motor deficit. Motor deficits that showed improvement in 36 months were considered transient, and motor deficits were considered permanent if they persisted after 36 months.

Data were imported into IBM SPSS ver. 20.1 (IBM Corp., Armonk, NY, USA) for analysis. Mann-Whitney test was used to analyze statistical differences between the two groups. Two-sided $p$-values $<0.05$ were considered statistically significant. The calculation of test operating characteristics (sensitivity and specificity) of the IONM was based on previous related studies [12,21,22]. Accordingly, IONM outcomes were classified in one of four ways. A true-positive event was defined as a signal degraded to warning criteria values, correlating with a postoperative neurologic deficit (whether or not the signal was recovered). A false-positive event was defined as a signal degraded to warning criteria values without postoperative neurologic deficit. The outcome was classified as true-negative when the signal remained consistent with baseline values, and the patient's postoperative neurologic status was unchanged. Finally, a false-negative event was defined as a signal that remained consistent with baseline values throughout a surgical procedure, but the patient demonstrated postoperative neurologic deficit.

\section{Results}

\section{Main results}

Significant IONM signal change occurred in $17.1 \%$ (11 of 64) of the patients (Table 2). Five patients had no postoperative neurologic deficits; another six patients $(9.3 \%)$ had a significant postoperative neurologic compromise. Of the six patients with postoperative impairments, three patients were able to walk, with motor grade 3 to 4 bilateral 


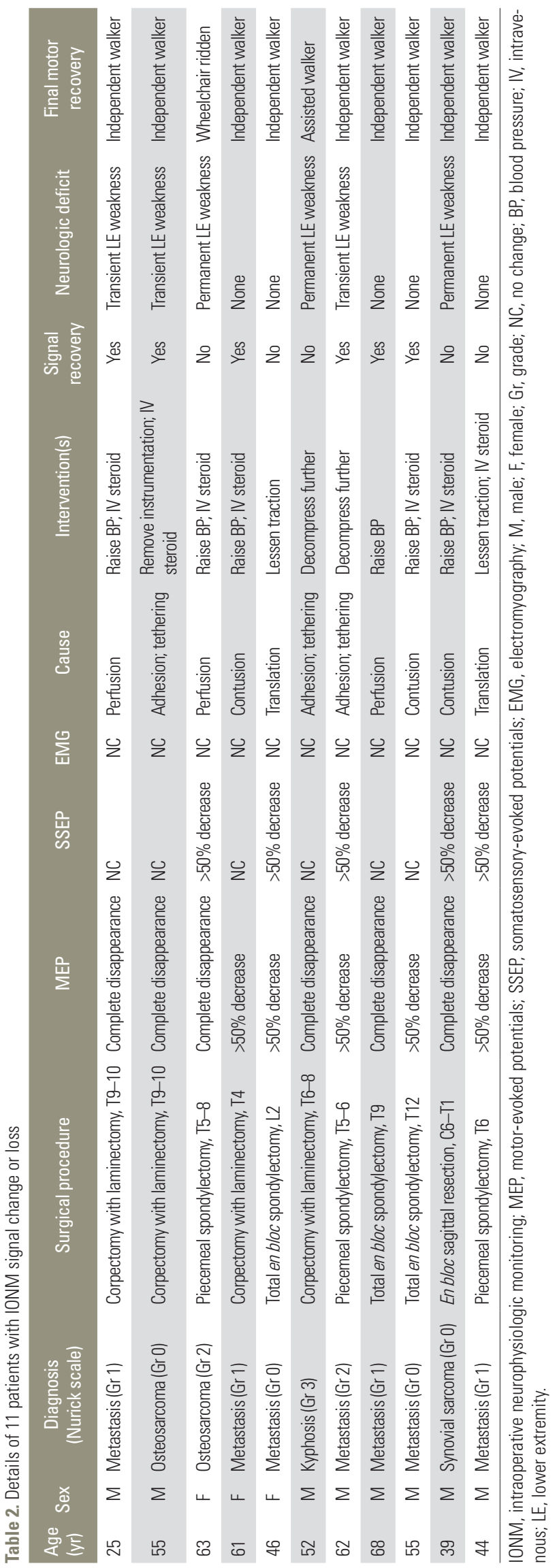

lower extremity weakness, and three patients were unable to walk, with bilateral lower extremity motor grade 1 . Of the three patients who lost walking ability postoperatively, one patient fully recovered at 18 months postoperatively, and the other two required a walking aid. Overall, permanent motor deficits were seen in three of the 64 patients (4.6\%) (Table 2). All neurologic deficits were from spinal cord dysfunction. The neurologic function did not deteriorate postoperatively in any patient who showed no significant IONM signal change.

The most frequent modality of IONM showing a significant signal change was MEP (100\%, 11 of 11), followed by SSEP $(45.4 \%, 5$ of 11$)$. No patient had only SSEP signal change, and all were combined with significant MEP change. In four patients, signal changes were not reversed at the end of the operation (irreversible-change group, 36.4\%) despite intervention by increasing the mean arterial pressure (MAP), performing further decompression, and/or checking patient and instrumentation positioning. In the remaining seven patients, the signal recovered above the warning criteria and remained there until the end of the operation (reversible-change group, 63.6\%) after appropriate interventions. The incidence of postoperative neurologic deficit was $42.8 \%$ ( 3 of 7 ) in the reversiblechange group and $75 \%$ (3 of 4 ) in the irreversible-change group. Permanent motor deficits occurred only in the irreversible-change group; interestingly, the reversiblechange group showed significant recovery of neurologic deficits during the follow-up period (Table 2).

Taken altogether, six of 11 patients showed both postoperative neurologic deficits and MEP changes. The sensitivity of MEP in predicting deficit was calculated at $100 \%$, and the specificity was measured at $91.4 \%$. For SSEP changes, the sensitivity was $83.3 \%$ and the specificity was $100 \%$. For the combined MEP and SSEP changes, the sensitivity was $100 \%$ and the specificity was $91.4 \%$. In three patients with permanent neurologic deficits, the sensitivity of the combined MEP and SSEP changes was the same (100\%), but the specificity was slightly lower (86.9\%) (Table 3).

The presumed causes of IONM signal change were as follows: adhesion/tethering $(n=3)$, translation $(n=2)$, perfusion ( $n=3)$, and contusion of the spinal cord $(n=3)$. The majority of them (8 of 11) were considered a mechanical insult to the spinal cord when the spinal column was totally destabilized or associated with severe epidural adhesion. However, three of them were not too related 
Table 3. Sensitivity and specificity of IONM in spine surgery involving three-column

\begin{tabular}{lcccc} 
& \multicolumn{2}{c}{ Postoperative motor deficit } & \multicolumn{2}{c}{ Permanent motor deficit } \\
\cline { 2 - 5 } & Sensitivity $(\%)$ & Specificity $(\%)$ & Sensitivity $(\%)$ & Specificity $(\%)$ \\
MEP & 100.0 & 91.4 & 100.0 & 86.9 \\
SSEP & 83.3 & 100.0 & 66.7 & 100.0 \\
\hline Combined & 100.0 & 91.4 & 100.0 & 86.9 \\
\hline
\end{tabular}

IONM, intraoperative neurophysiologic monitoring; MEP, motor-evoked potentials; SSEP, somatosensory-evoked potentials.

to mechanical insult and were thought to be related to compromised perfusion to the spinal cord according to excessive intraoperative bleeding or interrupting vascular supply to the spinal cord. The average MAP measured at the time of signal change of the patients presumed to have compromised perfusion of the spinal cord and the other patients were $45.7 \mathrm{~mm} \mathrm{Hg}$ and $68.5 \mathrm{~mm} \mathrm{Hg}$, respectively $(p<0.05)$. The average degree of MAP reduction in the two groups was $47.6 \%$ and $23.7 \%$, respectively $(p<0.05)$, compared to the MAP measured in a stable state during surgery. The average estimated blood loss (EBL) was 3,275 $\mathrm{mL}$ and $3,150 \mathrm{~mL}$, respectively $(p=1)$.

The following interventions were performed to reverse the data: raising blood pressure to at least $90 \mathrm{~mm} \mathrm{Hg}$ with blood transfusion, fluids, and/or vasopressors $(n=6)$, instrumentation removal $(\mathrm{n}=1)$, lessening traction $(\mathrm{n}=2)$, further decompression $(\mathrm{n}=2)$, and intravenous steroid administration $(\mathrm{n}=7)$.

Corpectomy with laminectomy was the most common interventional procedure that induced IONM signal change, which was done for the circumferential decompression of the spinal cord or the removal of metastatic spinal tumor $(45.5 \%, 5$ of 11$)$. However, based on the entire cohort, the incidence of signal change/loss in each procedure was lowest in corpectomy with laminectomy. It occurred in $25 \%$ ( 3 of 12 ) of the total en bloc-spondylectomy cases and $30 \%$ (3 of 10) of the piecemeal-spondylectomy cases. The three total en bloc-spondylectomy cases with IONM signal change had no neurologic change postoperatively.

Ten patients of the IONM signal change groups were diagnosed with either primary or metastatic spinal tumor. The remaining one patient had post-tuberculosis thoracolumbar kyphosis complicated with compressive myelopathy. Thus, almost all patients $(90.9 \%, 10$ of 11$)$ had tumorrelated problems (Table 2).

There were 19 patients with preoperative neurologic

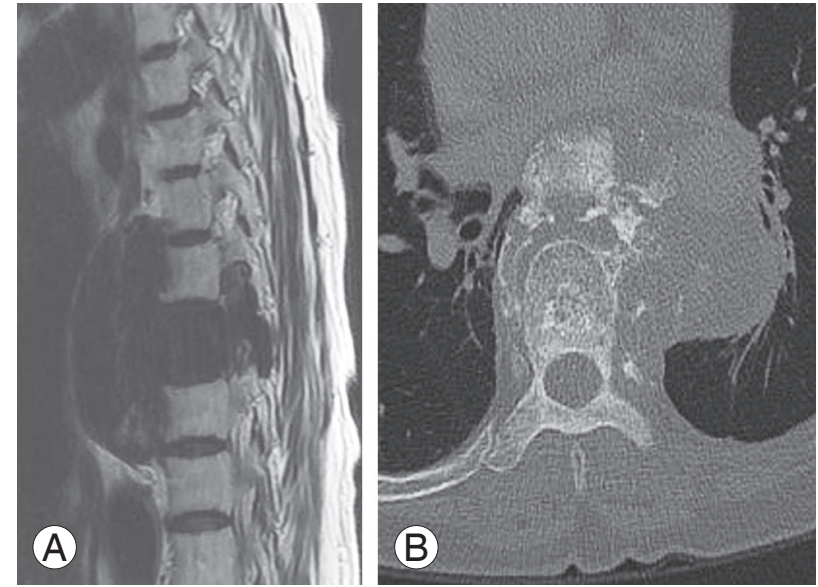

Fig. 1. (A) Sagittal T2 weighted magnetic resonance imaging revealed a dark bone lesion in T7, left epidural mass and paravertebral mass with left neural foraminal encroachment. (B) Axial chest computed tomography scan revealed an osteosclerotic bone lesion in $T 7$ with paravertebral mass abutting descending thoracic aorta, left atrium and pulmonary veins.

deficits (motor weakness or myelopathic gait), and three of them $(15.8 \%)$ had IONM signal change. Postoperative neurologic deficits occurred in two of three patients (66.6\%) with signal changes. Of the patients without preoperative neurologic deficits $(n=45)$, eight patients $(17.8 \%)$ had IONM signal change. Four of them (50\%) had postoperative neurologic deficits.

\section{Illustrative case}

A 63-year-old woman was diagnosed with osteosarcoma (Fig. 1). A posterior approach was performed first, and a second anterior approach with a thoracic surgeon was performed after 1 week. Preoperative neurologic examination was normal. Baseline MEP data were well-formed and reliable. During en bloc resection of the T7 posterior arch, bilateral lower extremity MEP signals were lost, and the amplitude of the left lower extremity SSEP was decreased by more than $50 \%$. There was no obvious impact 

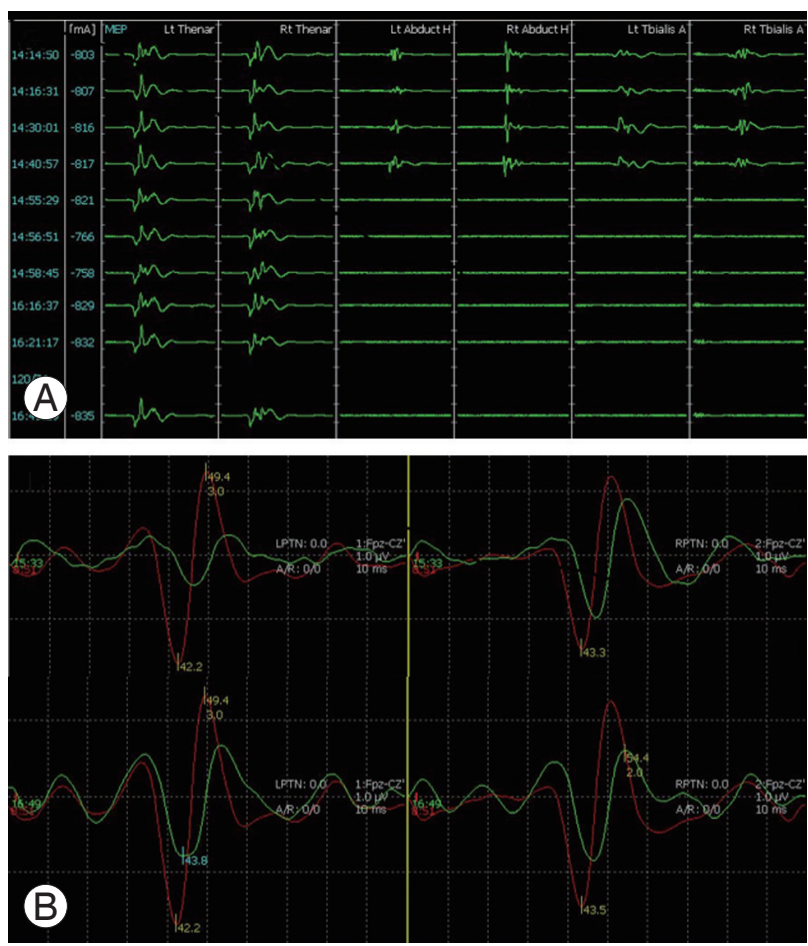

Fig. 2. (A) Acute drop of motor-evoked potentials amplitudes. (B) Top: amplitude of the left lower extremity somatosensory-evoked potentials (SSEP, green line) also decreased compared with the baseline data (red line); bottom: SSEP signal was recovered after increasing mean blood pressure. on the spinal cord, and there was a rapid blood pressure drop due to copious epidural bleeding during tumor tissue removal. At the time of signal loss, the patient's MAP was $36 \mathrm{~mm} \mathrm{Hg}$. It was reduced by $60 \%$ compared to stable blood pressure $(90 \mathrm{~mm} \mathrm{Hg}$ ) during surgery. After increasing the blood pressure, the SSEP signal recovered to $80 \%$ of baseline until the end of the surgery, but the MEP signal did not recover (Fig. 2). The patient's postoperative neurologic examination revealed global weakness (grade 1) of the bilateral lower extremity. One week later, the main mass with involved descending aorta was removed by an anterior approach in cooperation with the thoracic surgeon, and reconstruction with metal cage and anterior instrument fixation were performed (Fig. 3). The patient's motor function gradually recovered, and self-ambulation was possible without the aid 2 years after the surgery.

\section{Discussion}

This study specifically reviewed 64 patients who underwent three-column spinal surgery with IONM and observed significant signal changes in 11 patients. Six of the 11 patients had postoperative neurologic deficits. The sensitivity of the IONM signal change was $100 \%$, and the specificity was $91.4 \%$. These results redemonstrated its usefulness and are comparable to other similar stud-
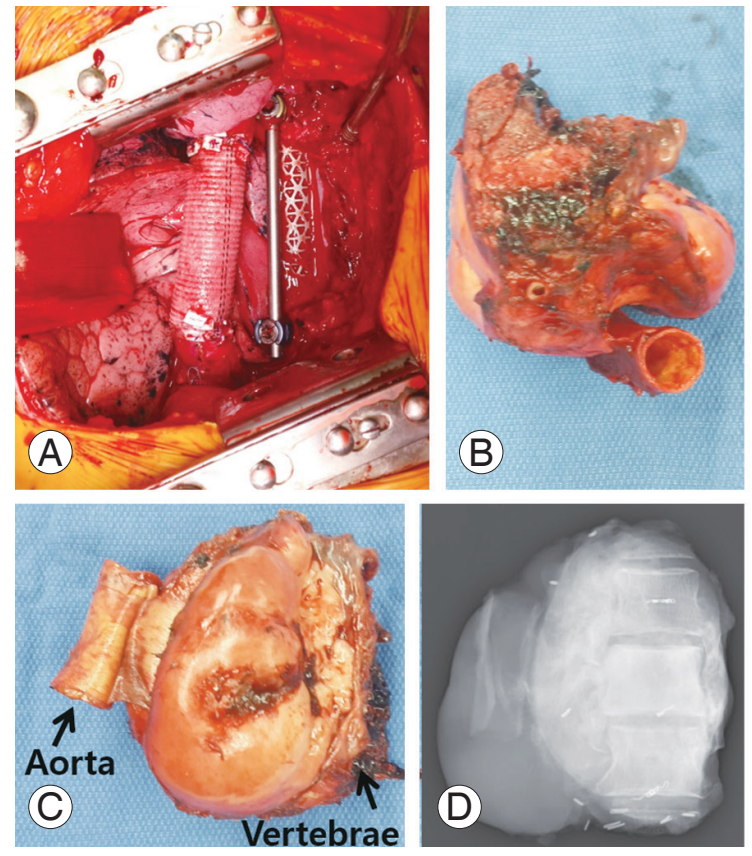
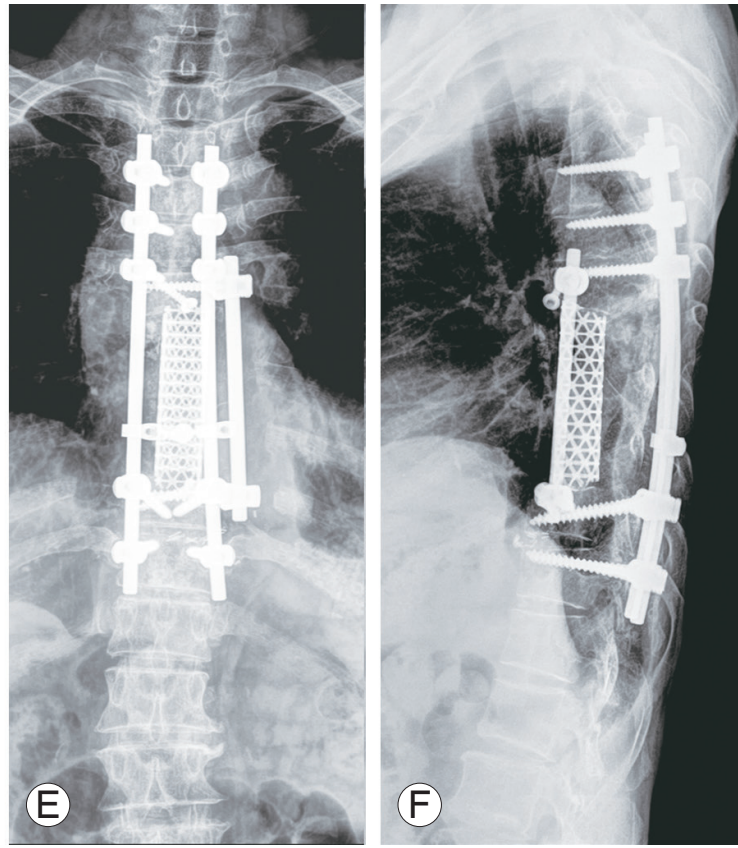

Fig. 3. (A-D) Tumor was resected, including T6, 7, 8 vertebral body and reconstructed with a woven vascular graft and metal cage, respectively. (E, F) Postoperative anteroposterior and lateral radiographs showing posterior instrumentation, T3-5, T9-10 and anterior cage, T6-8. 
ies reporting the usefulness of IONM in various spinal surgeries [19,20,23-25]. In addition, this study demonstrated surgical causes related to the significant IONM signal change. The IONM events usually occurred when the spinal column was totally destabilized during surgery, increasing the risk of mechanical insult, such as contusion, translation, or tethering with or without epidural adhesion. However, it also occurred without any recognizable mechanical insult, while compromise of perfusion to the spinal cord was suspected, especially in excessive intraoperative bleeding or diminished vascular supply of the spinal cord. Many studies have reported that MEP is a sensitive marker for impending neurologic deficit due to spinal cord ischemia during spinal surgery and emphasized maintaining adequate blood pressure during surgery $[12,21,26,27]$. The average MAP measured at the time of signal change/loss of the patients presumed to have compromised perfusion was $45.7 \mathrm{~mm} \mathrm{Hg}$, statistically significantly lower than other patients $(68.5 \mathrm{~mm} \mathrm{Hg}, p<0.05)$. However, there was no statistically significant difference in EBL during surgery $(p=1)$. Therefore, it can be argued that it is important to maintain proper blood pressure regardless of blood loss during surgery. It reinforces the opinion of previous related studies, and intraoperative MAP should be maintained to at least 60 to $65 \mathrm{~mm} \mathrm{Hg}$ to minimize neurologic deficit $[12,21,26]$.

In this study, 11 patients had significant signal changes that identified them as being at risk for neurologic injury during surgery. Immediate notification and close cooperation of the surgeon and the anesthesiologist, before permanent neurologic injury occurs, can result in adequate intervention, such as mechanical adjustment, administration of dexamethasone, and maintenance of adequate blood pressure. It is hard to prove the effectiveness of each intervention in each case; however, the IONM doubtlessly provides a motive to assess and avoid the potential cause of increasing neurologic complication risk by monitoring the neuroelectrophysiologic function of the spinal cord and detecting a significant change. In comparison to cases with the recovery of the IONM signal showing no permanent neurologic deficit, permanent neurologic deficits occurred in three patients, all with irreversible signal change until the end of the operation. Although it is not an absolute prognostic factor, recovery of the signal during surgery can be expected to have a favorable neurologic prognosis.

In this study, IONM signal change events were more common in tumor resection cases than deformity correction cases. Compared to $4.5 \%$ ( 1 of 22) of the patients without tumors, $23.8 \%$ (10 of 42 ) of the tumor patients had events and $14.3 \%$ ( 6 of 42 ) of the tumor patients had postoperative neurologic deficits (true-positive events). Several reasons were considered for the higher incidence rate of signal change or postoperative neurologic injury in tumor-related surgery. First, in surgical treatment for metastatic tumors, preoperative neurologic deficits are frequently present already, and there is often a high risk of neural tissue damage during tumor removal due to severe adhesion from preoperative radiation therapy. Second, in primary tumors where en bloc resection is usually performed with curative intention, significant structural instability is accompanied by the complete disconnection of the proximal and distal vertebral column to the segment of tumor location. Perfusion of the spinal cord is also likely diminished during circumferential dissection, $360^{\circ}$ around the tumor to secure surgical margin.

There are several limitations to this study. First, this study has a relatively small number of subjects and is retrospective. However, given the rarity of three-column spinal surgery, these numbers are large enough to be meaningful even in retrospect. Second, sensory deficits were not checked, and this study focused only on postoperative motor function. Third, IONM data measurement may not have been accurate in patients with preoperative paralysis, as MEP response amplitudes in weak muscles are lower and more highly variable [28]. In addition, only patients who had significant change or loss of IONM signal were analyzed. This is an initial trial, and a comparison study to a control group would be required to increase credibility.

\section{Conclusions}

This study encompasses three-column spinal surgeries from the cervical spine to the lumbar spine. There were 11 cases (17.1\%) of significant IONM signal change during surgery. The events usually occurred when the spinal column was totally destabilized during surgery in relation to mechanical insult such as contusion, translation, or tethering with or without epidural adhesion. However, it also occurred without recognizable mechanical insult, while compromise of perfusion to the spinal cord was suspected, especially in excessive intraoperative bleeding, or $360^{\circ}$ dissection of the spinal cord with devascularization. Based on the results of this study, to enhance neurologic safety 
in three-column spinal surgery, surgeons should pay attention to protect the spinal cord from mechanical insult, especially when the spinal column was totally destabilized during surgery, and not to compromise perfusion to the spinal cord in close cooperation with a neurologist and anesthesiologist.

\section{Conflict of Interest}

No potential conflict of interest relevant to this article was reported.

\section{References}

1. Cristante L, Herrmann HD. Surgical management of intramedullary spinal cord tumors: functional outcome and sources of morbidity. Neurosurgery 1994;35:69-74.

2. Bridwell KH, Lenke LG, Baldus C, Blanke K. Major intraoperative neurologic deficits in pediatric and adult spinal deformity patients: incidence and etiology at one institution. Spine (Phila Pa 1976) 1998;23:324-31.

3. Bilsky MH, Boland P, Lis E, Raizer JJ, Healey JH. Single-stage posterolateral transpedicle approach for spondylectomy, epidural decompression, and circumferential fusion of spinal metastases. Spine (Phila Pa 1976) 2000;25:2240-9.

4. Wang JC, Boland P, Mitra N, et al. Single-stage posterolateral transpedicular approach for resection of epidural metastatic spine tumors involving the vertebral body with circumferential reconstruction: results in 140 patients: invited submission from the Joint Section Meeting on Disorders of the Spine and Peripheral Nerves, March 2004. J Neurosurg Spine 2004;1:287-98.

5. Constantini S, Miller DC, Allen JC, Rorke LB, Freed D, Epstein FJ. Radical excision of intramedullary spinal cord tumors: surgical morbidity and longterm follow-up evaluation in 164 children and young adults. J Neurosurg 2000;93(2 Suppl):183-93.

6. Yang C, Zheng Z, Liu H, Wang J, Kim YJ, Cho S. Posterior vertebral column resection in spinal deformity: a systematic review. Eur Spine J 2016;25:2368-75.

7. Lenke LG, Newton PO, Sucato DJ, et al. Complications after 147 consecutive vertebral column resections for severe pediatric spinal deformity: a multi- center analysis. Spine (Phila Pa 1976) 2013;38:11932.

8. Wong YC, Chau WWJ, Kwok KO, Law SW. Incidence and risk factors for implant failure in spinal metastasis surgery. Asian Spine J 2020;14:878-85.

9. Kim GU, Chang MC, Kim TU, Lee GW. Diagnostic modality in spine disease: a review. Asian Spine J 2020;14:910-20.

10. Kumar N, Patel R, Tan BW, et al. Asymptomatic construct failure after metastatic spine tumor surgery: a new entity or a continuum with symptomatic failure? Asian Spine J 2021;15:636-49.

11. Fehlings MG, Brodke DS, Norvell DC, Dettori JR. The evidence for intraoperative neurophysiological monitoring in spine surgery: does it make a difference? Spine (Phila Pa 1976) 2010;35:S37-46.

12. Schwartz DM, Auerbach JD, Dormans JP, et al. Neurophysiological detection of impending spinal cord injury during scoliosis surgery. J Bone Joint Surg Am 2007;89:2440-9.

13. Rajappa D, Khan MM, Masapu D, et al. Multimodal intraoperative neurophysiological monitoring in spine surgeries: the experience at a spine centre through years. Asian Spine J 2020 Dec 30 [Epub]. https://doi.org/10.31616/asj.2020.0400.

14. Park P, Wang AC, Sangala JR, et al. Impact of multimodal intraoperative monitoring during correction of symptomatic cervical or cervicothoracic kyphosis. J Neurosurg Spine 2011;14:99-105.

15. Thuet ED, Padberg AM, Raynor BL, et al. Increased risk of postoperative neurologic deficit for spinal surgery patients with unobtainable intraoperative evoked potential data. Spine (Phila Pa 1976) 2005;30:2094-103.

16. Wilson-Holden TJ, Padberg AM, Lenke LG, Larson BJ, Bridwell KH, Bassett GS. Efficacy of intraoperative monitoring for pediatric patients with spinal cord pathology undergoing spinal deformity surgery. Spine (Phila Pa 1976) 1999;24:1685-92.

17. Decruz J, Kaliya-Perumal AK, Wong KH, Kumar DS, Yang EW, Oh JY. Neuromonitoring in cervical spine surgery: when is a signal drop clinically significant? Asian Spine J 2021;15:317-23.

18. Calancie B, Molano MR. Alarm criteria for motorevoked potentials: what's wrong with the "presenceor-absence" approach? Spine (Phila Pa 1976) 2008;33:406-14. 
19. Nuwer MR, Dawson EG, Carlson LG, Kanim LE, Sherman JE. Somatosensory evoked potential spinal cord monitoring reduces neurologic deficits after scoliosis surgery: results of a large multicenter survey. Electroencephalogr Clin Neurophysiol 1995;96:6-11.

20. Padberg AM, Wilson-Holden TJ, Lenke LG, Bridwell KH. Somatosensory- and motor-evoked potential monitoring without a wake-up test during idiopathic scoliosis surgery: an accepted standard of care. Spine (Phila Pa 1976) 1998;23:1392-400.

21. Langeloo DD, Lelivelt A, Louis Journee H, Slappendel R, de Kleuver M. Transcranial electrical motorevoked potential monitoring during surgery for spinal deformity: a study of 145 patients. Spine (Phila Pa 1976) 2003;28:1043-50.

22. Raynor BL, Bright JD, Lenke LG, et al. Significant change or loss of intraoperative monitoring data: a 25 -year experience in 12,375 spinal surgeries. Spine (Phila Pa 1976) 2013;38:E101-8.

23. Dawson EG, Sherman JE, Kanim LE, Nuwer MR. Spinal cord monitoring: results of the Scoliosis Research Society and the European Spinal Deformity Society survey. Spine (Phila Pa 1976) 1991;16(8 Suppl):S361-4.
24. Herdmann J, Deletis V, Edmonds HL Jr, Morota N. Spinal cord and nerve root monitoring in spine surgery and related procedures. Spine (Phila $\mathrm{Pa} 1976$ ) 1996;21:879-85.

25. Tobert DG, Glotzbecker MP, Hresko MT, et al. Efficacy of intraoperative neurophysiologic monitoring for pediatric cervical spine surgery. Spine (Phila $\mathrm{Pa}$ 1976) 2017;42:974-8.

26. Pastorelli F, Di Silvestre M, Plasmati R, et al. The prevention of neural complications in the surgical treatment of scoliosis: the role of the neurophysiological intraoperative monitoring. Eur Spine J 2011;20 Suppl 1:S105-14.

27. Pelosi L, Lamb J, Grevitt M, Mehdian SM, Webb JK, Blumhardt LD. Combined monitoring of motor and somatosensory evoked potentials in orthopaedic spinal surgery. Clin Neurophysiol 2002;113:1082-91.

28. Lyon R, Feiner J, Lieberman JA. Progressive suppression of motor evoked potentials during general anesthesia: the phenomenon of "anesthetic fade". J Neurosurg Anesthesiol 2005;17:13-9. 